Geometry $8 \mathcal{T}$ Topology

Volume 8 (2004) 277-293

Published: 14 February 2004

To Ada

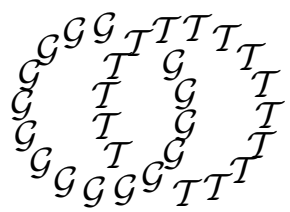

\title{
A few remarks about symplectic filling
}

\author{
YAKOV ELIASHBERG \\ Department of Mathematics, Stanford University \\ Stanford CA 94305-2125, USA \\ Email: eliash@gauss.stanford.edu
}

\begin{abstract}
We show that any compact symplectic manifold $(W, \omega)$ with boundary embeds as a domain into a closed symplectic manifold, provided that there exists a contact plane $\xi$ on $\partial W$ which is weakly compatible with $\omega$, i.e. the restriction $\left.\omega\right|_{\xi}$ does not vanish and the contact orientation of $\partial W$ and its orientation as the boundary of the symplectic manifold $W$ coincide. This result provides a useful tool for new applications by Ozsváth-Szabó of Seiberg-Witten Floer homology theories in three-dimensional topology and has helped complete the Kronheimer-Mrowka proof of Property P for knots.
\end{abstract}

\section{AMS Classification numbers Primary: 53C15}

Secondary: 57M50

Keywords: Contact manifold, symplectic filling, symplectic Lefschetz fibration, open book decomposition

Proposed: Leonid Polterovich

Seconded: Peter Ozsváth, Dieter Kotschick
Received: 25 November 2003

Revised: 13 January 2004 


\section{Introduction}

All manifolds which we consider in this article are assumed oriented. A contact manifold $V$ of dimension three carries a canonical orientation. In this case we will denote by $-V$ the contact manifold with the opposite orientation. Contact plane fields are assumed co-oriented, and therefore oriented. Symplectic manifolds are canonically oriented, and so are their boundaries.

We prove in this article the following theorem:

Theorem 1.1 Let $(V, \xi)$ be a contact manifold and $\omega$ a closed 2-form on $V$ such that $\left.\omega\right|_{\xi}>0$. Suppose that we are given an open book decomposition of $V$ with a binding $B$. Let $V^{\prime}$ be obtained from $V$ by a Morse surgery along $B$ with a canonical 0-framing, so that $V^{\prime}$ is fibered over $S^{1}$. Let $W$ be the corresponding cobordism, $\partial W=(-V) \cup V^{\prime}$. Then $W$ admits a symplectic form $\Omega$ such that $\left.\Omega\right|_{V}=\omega$ and $\Omega$ is positive on fibers of the fibration $V^{\prime} \rightarrow S^{1}$.

Remark 1.2 Note that the binding $B$ has a canonical decomposition of its tubular neighborhood given by the pages of the book. The 0 -surgery along $B$ is the Morse surgery associated with this decomposition. If the binding is disconnected then we assume that the surgery is performed simultaneously along all the components of $B$.

We will deduce the following result from Theorem 1.1

Theorem 1.3 Let $(V, \xi)$ and $\omega$ be as in Theorem 1.1. Then there exists a symplectic manifold $\left(W^{\prime}, \Omega^{\prime}\right)$ such that $\partial W^{\prime}=-V$ and $\left.\Omega^{\prime}\right|_{V}=\omega$. Moreover, one can arrange that $H_{1}\left(W^{\prime}\right)=01$ and that $\left(W^{\prime}, \Omega^{\prime}\right)$ contains the symplectic cobordism $(W, \Omega)$ constructed in Theorem 1.1 as a subdomain adjacent to the boundary. In particular, any symplectic manifold which weakly fills (see Section 4 below) the contact manifold $(V, \xi)$ can be symplectically embedded as a subdomain into a closed symplectic manifold.

Corollary 1.4 Any weakly (resp. strongly) semi-fillable (see [10]) contact manifold is weakly (resp. strongly) fillable.

Remark 1.5 Theorem 1.1 serves as a missing ingredient in proving that the Ozsváth-Szabó contact invariant $c(\xi)$ does not vanish for weakly symplectically fillable (and hence for non-existing anymore semi-fillable) contact structures.

\footnotetext{
${ }^{1}$ This observation is due to Kronheimer and Mrowka, see [20].
} 
This and other applications of the results of this article in the Heegaard Floer homology theory are discussed in the paper of Peter Ozsváth and Zoltán Szabó, see [27]. The observation made in this paper also helped to streamline the program of Peter Kronheimer and Tomasz Mrowka for proving the Property $\mathrm{P}$ for knots, see their paper [20].

Acknowledgements This article is my answer to the question I was asked by Olga Plamenevskaya and David Gay during the workshop on Floer homology for 3-manifolds in Banff International Research Station. I want to thank them, as well as Selman Akbulut, Michael Freedman, Robion Kirby, Peter Kronheimer, Robert Lipshitz, Tomasz Mrowka, Peter Ozsváth, Michael Sullivan and Zoltán Szabó for stimulating discussions, and Augustin Banyaga, Steven Kerckhoff, Leonid Polterovich and András Stipsicz for providing me with the necessary information. I am grateful to John Etnyre, Tomasz Mrowka, András Stipsicz and Leonid Polterovich for their critical remarks concerning a preliminary version of this paper. I also want to thank Peter Kronheimer and Tom Mrowka with sharing with me their alternative "flux-fixing argument" (see Lemma 3.4 below).

This research was partially supported by NSF grants DMS-0204603 and DMS0244663 .

\section{Proof of Theorem 1.1}

We begin with the following lemma which is a slight reformulation of Proposition 3.1 in [6]. A similar statement is contained also in [19].

Lemma 2.1 Let $(V, \xi)$ and $\omega$ be as in Theorem 1.1. Then given any contact form $\alpha$ for $\xi$ and any $C>0$ one can find a symplectic form $\Omega$ on $V \times[0,1]$ such that

a) $\left.\Omega\right|_{V \times 0}=\omega ;$

b) $\Omega_{V \times[1-\varepsilon, 1]}=\omega+C d(t \alpha)$, where $t \in[1-\varepsilon, 1]$ and $0<\varepsilon<1$;

c) $\Omega$ induces the negative orientation on $V \times 0$ and positive on $V \times 1$.

Proof By assumption

$$
\left.\omega\right|_{\xi}=\left.f d \alpha\right|_{\xi}=\left.d(f \alpha)\right|_{\xi}
$$


for a positive function $V \rightarrow \mathbb{R}$. Set $\tilde{\alpha}=f \alpha$. Then $\omega=d \tilde{\alpha}+\tilde{\alpha} \wedge \beta$. Take a smooth function $h: V \times[0,1] \rightarrow \mathbb{R}$ such that

$$
\left.h\right|_{V \times 0}=0,\left.h\right|_{V \times[1-\varepsilon, 1]}=\frac{C t}{f}, \frac{d h}{d t}>0,
$$

where $t$ is the coordinate corresponding to the projection $V \times[0,1] \rightarrow[0,1]$. Consider the form $\Omega=\omega+d(h \tilde{\alpha})$. Here we keep the notation $\omega$ and $\tilde{\alpha}$ for the pull-backs of $\omega$ and $\tilde{\alpha}$ to $V \times[0,1]$. Then we have

$$
\Omega=d \tilde{\alpha}+\tilde{\alpha} \wedge \beta+d_{V} h \wedge \tilde{\alpha}+\frac{d h}{d t} d t \wedge \tilde{\alpha},
$$

where $d_{V} h$ denotes the differential of $h$ along $V$. Then

$$
\Omega \wedge \Omega=2 \frac{d h}{d t} d t \wedge \tilde{\alpha} \wedge d \tilde{\alpha}>0 .
$$

Hence $\Omega$ is symplectic and it clearly satisfies the conditions a) - c).

Let us recall that a contact form $\lambda$ on $V$ is called compatible with the given open book decomposition (see [14]) if

a) there exists a neighborhood $U$ of the binding $B$, and the coordinates $(r, \varphi, u) \in[0, R] \times \mathbb{R} / 2 \pi \mathbb{Z} \times \mathbb{R} / 2 \pi \mathbb{Z}$ such that

$$
U=\{r \leq R\} \text { and }\left.\lambda\right|_{U}=h(r)\left(d u+r^{2} d \varphi\right),
$$

where the positive $C^{\infty}$-function $h$ satisfies the conditions

$$
h(r)-h(0)=-r^{2} \text { near } r=0 \text { and } h^{\prime}(r)<0 \text { for all } r>0 ;
$$

b) the parts of pages of the book in $U$ are given by equations $\varphi=$ const;

c) $d \alpha$ does not vanish on the pages of the book (with the binding deleted).

Remark 2.2 An admissible contact form $\alpha$ defines an orientation of pages and hence an orientation of the binding $B$ as the boundary of a page. On the other hand, the form $\alpha$ defines a co-orientation of the contact plane field, and hence an orientation of $B$ as a transversal curve. These two orientations of $B$ coincide.

Remark 2.3 By varying admissible forms for a given contact plane field one can arrange any function $h$ with the properties described in a). Indeed, suppose we are given another function $\widetilde{h}$ which satisfies a). We can assume without loss of generality that $\alpha$ has the presentation a) on a bigger domain $U^{\prime}=\left\{r \leq R^{\prime}\right\}$ for $R^{\prime}>R$. Let us choose $c>0$ such that $\widetilde{h}(R)>\operatorname{ch}(R)$ and extend $\widetilde{h}$ to $\left[0, R^{\prime}\right]$ in such a way that $\widetilde{h}^{\prime}(r)<0$ and $\widetilde{h}(r)=\operatorname{ch}(r)$ near $R^{\prime}$. Then the form $\widetilde{h} \alpha$ on $U^{\prime}$ extended to the rest of the manifold $V$ as $c \alpha$ is admissible for the given open book decomposition. 
Now we are ready to prove Theorem 1.1

Proof of Theorem 1.1 Take a constant $a>0$ and consider a smooth on $[0,1)$ function $g:[0,1] \rightarrow \mathbb{R}$ such that $\left.g\right|_{[0,1 / 2]}=a, g(t)=\sqrt{1-t^{2}}$ for $t$ near 1 and $g^{\prime}<0$ on $(1 / 2,1)$.

In the standard symplectic $\mathbb{R}^{4}$ which we identify with $\mathbb{C}^{2}$ with coordinates $\left(z_{1}=r_{1} e^{i \varphi_{1}}=x_{1}+i y_{1}, z_{2}=r_{2} e^{i \varphi_{2}}=x_{2}+i y_{2}\right)$ let us consider a domain

$$
\widetilde{P}=\left\{r_{1} \leq g\left(r_{2}\right), r_{2} \in[0,1]\right\} .
$$

The domain $\widetilde{P}$ is contained in the polydisc $P=\left\{r_{1} \leq a, r_{2} \leq 1\right\}$ and can be viewed as obtained by smoothing the corners of $P$.

Let us denote by $\Gamma$ the part of the boundary of $\widetilde{P}$ given by

$$
\Gamma=\left\{\left\{r_{1}=g\left(r_{2}\right), r_{1} \in[1 / 2,1]\right\} .\right.
$$

Note that $\Gamma$ is $C^{\infty}$-tangent to $\partial P$ near its boundary. The primitive

$$
\gamma=\frac{1}{2}\left(r_{1}^{2} d \varphi_{1}+r_{2}^{2} d \varphi_{2}\right)
$$

of the standard symplectic form

$$
\omega_{0}=d x_{1} \wedge d y_{1}+d x_{2} \wedge d y_{2}=r_{1} d r_{1} \wedge d \varphi_{1}+r_{2} d r_{2} \wedge d \varphi_{2}
$$

restricts to $\Gamma$ as a contact form

$$
\left.\gamma\right|_{\Gamma}=\frac{r_{2}^{2}}{2}\left(\frac{g^{2}\left(r_{2}\right)}{r_{2}^{2}} d \varphi_{1}+d \varphi_{2}\right) .
$$

Consider the product $G=S^{2} \times \mathrm{D}^{2}$ with the split symplectic structure $\omega_{0}=$ $\sigma_{1} \oplus \sigma_{2}$, where the total area of the form $\sigma_{1}$ on $S^{2}$ is equal to $2 \pi$ and the total area of the form $\sigma_{2}$ on the disc $D^{2}$ is equal to $\pi a^{2}$. Note that if $S_{+}^{2}$ and $S_{-}^{2}$ denote the upper and lower hemispheres of $S^{2}$ of equal area, then there exists a symplectomorphism

$$
\Phi: P \rightarrow S_{+}^{2} \times D^{2} \subset S^{2} \times D^{2}=G .
$$

Let $H$ be the closure of $G \backslash \Phi(\widetilde{P})$ and $\widetilde{\Gamma}$ denote the image $\Phi(\Gamma) \subset \partial H$. Note that

$$
\Delta=\overline{\partial H \backslash \Gamma}=\widetilde{S}_{-}^{2} \times \partial D^{2}=\bigcup_{x \in \partial D^{2}} \widetilde{S}_{-}^{2} \times x=\bigcup_{x \in \partial D^{2}} D_{x},
$$

where $\widetilde{S}_{-}^{2}, S_{-}^{2} \subset \widetilde{S}_{-}^{2} \subset S^{2}$, is a disc of area $\frac{9 \pi}{4}$. Thus $H$ is a 2 -handle whose boundary $\partial H$ consists of $\Gamma$ and $\Delta$ which meets along an infinitely sharp corner $\Gamma \cap \Delta$. The part $\Delta$ is fibered by discs $D_{x}, x \in \partial D^{2}$, which are symplectic with respect to the form $\omega_{0}$. 
Consider now a contact form $\lambda$ on $V$ compatible with the given open book decomposition. In particular, on a neighborhood

$$
U=[0, R] \times \mathbb{R} / 2 \pi \mathbb{Z} \times \mathbb{R} / 2 \pi \mathbb{Z} \subset V
$$

of the binding $B$ we have $\left.\lambda\right|_{U}=h(r)\left(d u+r^{2} d \varphi\right)$, where the positive $C^{\infty}$ function $h$ satisfies the conditions

$$
h(r)-h(0)=-r^{2} \text { near } r=0 \text { and } h^{\prime}(r)<0 \text { for all } r>0 .
$$

Let us choose $a=\frac{R}{2}$, and consider a diffeomorphism $F: \Gamma \rightarrow U$ given by the formula

$$
r=\frac{g\left(r_{2}\right)}{r_{2}}, \varphi=\varphi_{1}, u=\varphi_{2}
$$

The function

$$
r_{2} \mapsto \frac{g\left(r_{2}\right)}{r_{2}}
$$

maps $\left[1, \frac{1}{2}\right]$ onto $[0,2 a]=[0, R]$. Let $\psi:[0, R] \rightarrow\left[1, \frac{1}{2}\right]$ be the inverse function. Then

$$
F_{*} \gamma=\frac{\psi^{2}(r)}{2}\left(r^{2} d \varphi+d u\right) .
$$

Hence $F$ is a contactomorphism

$$
(\Gamma,\{\gamma=0\}) \rightarrow(U,\{\lambda=0\}=\xi) .
$$

Moreover, the form $F_{*} \gamma$, extended to $V$ as $\lambda$ on $V \backslash U$, defines on $V$ a smooth contact form compatible with the given open book decomposition.

Now we use Lemma 2.1 to define on the collar $V \times[0,1]$ a symplectic form $\Omega$ which satisfies the conditions $[2.1 \mathrm{a})-\mathrm{c}$ ), where the constant $C$ will be chosen later. In particular, near $V \times 1$ we have $\Omega=C d t \lambda+\omega$. Viewing $\Gamma$ as a part of the boundary of the handle $H$, we can extend $F$ to a symplectomorphism, still denoted by $F$, of a neighborhood of $\Gamma \subset H$ endowed with the standard symplectic structure $C \omega_{0}$ to a neighborhood of $U \subset V=V \times 1$ in $V \times[0,1]$ endowed with the symplectic structure $C d(t \lambda)$. Note that the closed form $F^{*} \omega$ is exact:

$$
F^{*} \omega=d \theta,
$$

and hence it extends to $H$ as $\widetilde{\omega}=d(\sigma \theta)$ where $\sigma$ is a cut-off function equal to 0 outside a neighborhood of $\Gamma$ in $H$. If $C$ is chosen sufficiently large then the form $\Omega_{0}=C \omega_{0}+\widetilde{\omega}$ is symplectic, and its restrictions to the discs $D_{x} \subset \Delta$, $x \in \partial D^{2}$, are symplectic as well. Hence the map $F$ can be used for attaching the symplectic handle $\left(H, C \omega_{0}+\widetilde{\omega}\right)$ to $V \times[0,1]$ along $U$. The resulted symplectic manifold

$$
W=V \times[0,1] \underset{U=F(\Gamma)}{\cup} H
$$


is the required symplectic cobordism. Indeed we have

$$
\partial\left(W, \Omega_{0}\right)=(-V, \omega) \cup\left(V^{\prime}, \omega^{\prime}\right),
$$

where the component $V^{\prime}$ of its boundary is fibered over $S^{1}$ by closed surfaces formed by parts of pages of the book inside $V \backslash U$ and discs $D_{x}$. These surfaces are symplectic with respect to the form $\omega^{\prime}=\left.\Omega_{0}\right|_{V^{\prime}}$.

\section{$3 \quad$ Filling of symplectic fibrations over circle}

A pair $(V, \omega)$, where $V$ is an oriented 3 -manifold fibered over $S^{1}=\mathbb{R} / \mathbb{Z}$, and $\omega$ is a closed 2-form which is positive on the fibers of the fibration, will be referred to as a symplectic fibration over $S^{1}$. The projection $V \rightarrow S^{1}$ will be denoted by $\pi$. We will assume that all symplectic fibrations we consider are normalized by the condition that the integral of $\omega$ over a fiber is equal to 1 . The form $\omega$ induces a 1 -dimensional characteristic foliation $\mathcal{F}_{\omega}$ on $V$ generated by the kernel of $\omega$. This foliation is transversal to the fibers of the fibration. The orientation of $V$ together with the symplectic orientation of the fibers defines an orientation of $\mathcal{F}_{\omega}$. Fixing a fiber $F_{0}$ over $0 \in S^{1}=\mathbb{R} / \mathbb{Z}$ we can define the holonomy diffeomorphism $\mathrm{Hol}_{V, \omega}: F_{0} \rightarrow F_{0}$. This is an area preserving diffeomorphism which defines $(V, \omega)$ uniquely up to a fiber preserving diffeomorphism fixed on $F_{0}$. Note that $\mathrm{Hol}_{-V, \omega}=\mathrm{Hol}_{V, \omega}^{-1}$. Two symplectic fibrations are equivalent via an equivalence fixed on $F_{0}$ if and only if their holonomy diffeomorphisms coincide. If for symplectic fibrations $\left(V, \omega_{0}\right)$ and $\left(V, \omega_{1}\right)$ the holonomy diffeomorphisms $\mathrm{Hol}_{V, \omega_{0}}$ and $\mathrm{Hol}_{V, \omega_{1}}$ are symplectically (resp. Hamiltonian) isotopic then $\left(V, \omega_{0}\right)$ and $\left(V, \omega_{1}\right)$ are called isotopic (resp. Hamiltonian isotopic). For a fixed smooth fibration $V \rightarrow S^{1}$ the isotopy between $\left(V, \omega_{0}\right)$ and $\left(V, \omega_{1}\right)$ is equivalent to a homotopy of forms $\omega_{0}$ and $\omega_{1}$ through closed forms positive on fibers of the fibration.

We will prove in this section

Theorem 3.1 For any symplectic fibration $(V, \omega)$ over $S^{1}$ there exists a compact symplectic 4-manifold $(W, \Omega)$ with

$$
\partial(W, \Omega)=(V, \omega) .
$$

One can additionally arrange that $H_{1}(W ; \mathbb{Z})=02$

The first ingredient in the proof in the following theorem of Akbulut and Ozbagci.

\footnotetext{
${ }^{2}$ This was observed by Kronheimer and Mrowka, see 20.
} 
Theorem 3.2 (See 2], Theorem 2.1) Theorem 3.1 holds up to homotopy. More precise, for any symplectic fibration $(V, \omega)$ as above there exists a compact symplectic 4-manifold $(W, \Omega)$ with $H_{1}(W)=0$ which has a structure of a symplectic Lefschetz fibration over $D^{2}$ and which restricts to $S^{1}=\partial D^{2}$ as a symplectic fibration $(V, \widetilde{\omega})$ homotopic to $(V, \omega)$.

The proof of this theorem is based on an observation (which the authors said they learned from Ivan Smith) that any element of the mapping class group of a closed surface can be presented as a composition of positive Dehn twists 3 W P Thurston's construction (see [28]) of symplectic structure on surface fibrations, and its adaptation by RE Gompf (see [15]) for Lefschetz fibrations with positive Dehn twists around exceptional fibers. Exploring the freedom of the construction one can arrange that $H_{1}(W ; \mathbb{Z})=0$. Indeed, for a Lefschetz fibration over $D^{2}$ we have $H_{1}(W) \simeq H_{1}\left(F_{0}\right) / C$, where $C \subset H_{1}\left(F_{0}\right)$ is the subgroup generated by the vanishing cycles. But the already mentioned above fact that the mapping class group is generated as a monoid by positive Dehn twists allows us to make any cycle in $H_{1}\left(F_{0}\right)$ vanishing.

The second ingredient in the proof of Theorem 3.1 is the following proposition based on a variation of an argument presented in 20, see Lemma 3.4 below.

Proposition 3.3 Let $(W, \Omega)$ be a Lefschetz fibration over $D^{2}$ and $(V, \omega)$ a symplectic fibration over $S^{1}$ which bounds it, $\partial(W, \Omega)=(V, \widetilde{\omega})$. Then for any symplectic fibration $(V, \widetilde{\omega})$ homotopic to $(V, \omega)$ there exists a symplectic form $\widetilde{\Omega}$ on $W$ such that $\partial(W, \widetilde{\Omega})=(V, \widetilde{\widetilde{\omega}})$ where $(V, \widetilde{\widetilde{\omega}})$ is Hamiltonian isotopic to $(V, \omega)$.

In other words, Proposition 3.3 together with Theorem 3.2 imply Theorem 3.1 up to Hamiltonian isotopy. Before proceeding with the proof we recall some standard facts about the flux homomorphism.

Let $F_{0}$ be a closed oriented surface of genus $g$ with an area form $\omega$. We denote by $\mathcal{D}=\mathcal{D}\left(F_{0}\right)$ the group of area preserving diffeomorphisms of $F_{0}$ and by $\mathcal{D}_{0}$ its identity component. The Lie algebra of $\mathcal{D}_{0}$ consists of symplectic vector fields, i.e. the vector fields, $\omega$-dual to closed forms. Hence, given an isotopy

\footnotetext{
${ }^{3}$ Here is a simple argument due to Peter Kronheimer which shows this. Take any generic genuine (i.e. having exceptional fibers) Lefschetz fibration over $C P^{1}$ with the fiber of prescribed genus. Then the product of +1 -twists corresponding to vanishing cycles is the identity. Therefore, a -1 -twist (and hence any -1 -twist) is a product +1 -twists.
} 
$f_{t} \in \mathcal{D}_{0}$ which connects $f_{0}=$ Id with $f_{1}=f$ then for the time-dependent vector field $v_{t}$ which generates $f_{t}$, i.e.

$$
v_{t}\left(f_{t}(x)\right)=\frac{d f_{t}(x)}{d t}, t \in[0,1], x \in F_{0},
$$

the form $\left.\alpha_{t}=v_{t}\right\lrcorner \omega$ is closed for all $t \in[0,1]$. Diffeomorphisms generated by time-dependent vector fields (11) dual to exact 1 -forms form a subgroup $\mathcal{D}_{H}$ of Hamiltonian diffeomorphisms. This subgroup is the kernel of a flux, or Calabi homomorphism (see [5]) which is defined as follows. Given $v_{t}$ generating $f$ as in (11) as its time-one map, we define

$$
\left.\operatorname{Flux}(f)=\int_{0}^{1}\left[v_{t}\right\lrcorner \sigma\right] d t
$$

where $\left.\left[v_{t}\right\lrcorner \sigma\right] \in H^{1}\left(F_{0} ; \mathbb{R}\right)$ is the cohomology class of the closed form $\left.v_{t}\right\lrcorner \sigma$. Though $\operatorname{Flux}(f)$, as defined by the above formula, is independent of the choice of the path $f_{t}$ up to homotopy, it may depend on the homotopy class of this path. Note, however, that when the genus of $F_{0}$ is $>1$ then $\mathcal{D}_{0}$ is contractible, and hence $\operatorname{Flux}(f)$ is well defined as an element of $H^{1}\left(F_{0} ; \mathbb{R}\right)$. If $F_{0}$ is the torus then $\operatorname{Flux}(f)$ is defined only modulo the total area of the torus, and hence it can be viewed as an element of $H^{1}\left(F_{0} ; \mathbb{R} / \mathbb{Z}\right)$. According to $[\underline{5}$,

$$
\mathcal{D}_{H}=\text { KerFlux, }
$$

i.e. two diffeomorphisms $f, g \in \mathcal{D}$ are Hamiltonian isotopic if and only if $\operatorname{Flux}\left(f \circ g^{-1}\right)=0 \underline{4}$

Therefore, Proposition 3.3 is equivalent to

Lemma 3.4 Suppose $(V, \omega)$ is a symplectic fibration over $S^{1}$ and $(W, \Omega)$ is a symplectic Lefschetz fibration over $D^{2}$ such that $\partial(W, \Omega)=(V, \omega)$ and $H_{1}(W)=0$. Then for any $a \in H^{1}\left(F_{0} ; \mathbb{R}\right)$ (or $H^{1}\left(F_{0} ; \mathbb{R} / \mathbb{Z}\right)$ if $F_{0}$ is the torus) there exists a symplectic form $\widetilde{\Omega}$ on $W$ such that

$$
\operatorname{Flux}\left(\operatorname{Hol}_{V, \omega} \circ \mathrm{Hol}_{V, \widetilde{\omega}}^{-1}\right)=a,
$$

where $\widetilde{\omega}=\left.\widetilde{\Omega}\right|_{V}$.

\footnotetext{
${ }^{4}$ This can be verified as follows. Let $f_{t} \in \mathcal{D}_{0}, t \in[0,1]$, be any symplectic isotopy connecting $f_{0}=f$ and $f_{1}=g$. Denote $a_{t}=\operatorname{Flux}\left(f_{t}\right)$ and choose a harmonic (for some metric) 1 -form $\alpha_{t}$ representing $a_{t} \in H^{1}\left(F_{0} ; \mathbb{R}\right)$. Set $\beta_{t}=\alpha_{t}-\alpha_{0}$. By assumption we have $\beta_{1}=0$. Let $\varphi_{t}$ be the time-one map of the symplectic flow generated by the symplectic vector field $v_{t} \omega$-dual to $\alpha_{t}$. Then for all $t \in[0,1]$ we have $\operatorname{Flux}\left(\varphi_{t}^{-1} \circ f_{t}\right)=$ $a_{0}$, and hence $\varphi_{t}^{-1} \circ f_{t}$ is a Hamiltonian isotopy between $f$ and $g$.
} 
Proof Let us recall that given an embedded path $\delta$ from a critical value $p \in$ $D^{2}$ of the Lefschetz fibration to a boundary point $q \in \partial D^{2}$ there exists a Lagrangian disc $\Delta_{\delta}$, called thimble, which projects to the path $\delta$ and whose boundary $\partial \Delta_{\delta} \subset F_{q}=\pi^{-1}(q)$ is a vanishing cycle. This thimble is formed by leaves of the characteristic foliation of the form $\left.\Omega\right|_{\pi^{-1}(\delta)}$ emanating from the corresponding critical point. Let us choose disjoint embedded paths $\delta_{1}, \ldots, \delta_{N}$ from all critical values of the Lefschetz fibration to points inside the arc $l=$ $[0,1 / 2] \subset \mathbb{R} / \mathbb{Z}=\partial D^{2}$. Let $\Delta_{i}=\Delta_{\delta_{i}}$ and $\gamma_{i}=\partial \Delta_{i}, i=1, \ldots, N$, be the corresponding thimbles and vanishing cycles. Using characteristics of $\omega$ as horizontal lines we can trivialize the fibration $V_{1 / 2}=\pi^{-1}(l) \rightarrow l$. Note that the inclusion $H_{1}\left(F_{0}\right) \rightarrow H_{1}(W)$ is surjective, and the kernel of this map is generated by the vanishing cycles (independently of paths along which they are transported to $F_{0}$ from a critical point). By the assumption, we have $H_{1}(W)=$ 0 and hence the projections of $\gamma_{i}$ to $F_{0}$ generate $H_{1}\left(F_{0}\right)$. Then the cohomology classes $D \gamma_{i} \in H^{1}\left(F_{0}\right), i=1, \ldots, N$, Poincaré dual to $\left[\gamma_{i}\right] \in H_{1}\left(F_{0}\right)$, generate $H^{1}\left(F_{0}\right)$. In particular, we can write

$$
a=\sum_{1}^{N} a_{i} D \gamma_{i} .
$$

Let us recall that there exists a neighborhood $U_{i}$ of $\Delta_{i}$ symplectomorphic to a disc bundle in $T^{*}\left(\Delta_{i}\right)$. Let $\left(q_{1}, q_{2}, p_{1}, p_{2}\right)$ be the canonical coordinates in $T^{*}\left(\Delta_{i}\right)$ such that

$$
\left.\Omega\right|_{\Delta_{i}}=d p_{1} \wedge d q_{1}+d p_{2} \wedge d q_{2}, \Delta_{i} \subset\left\{p_{1}=p_{2}=0\right\}
$$

and $U_{i}=\left\{\|p\|^{2}=p_{1}^{2}+p_{2}^{2}<\varepsilon^{2}\right\}$. Let $\sigma:[0, \varepsilon] \rightarrow \mathbb{R}$ be a non-negative function constant near 0 and equal to 0 near $\varepsilon$ and such that

$$
\int_{\|p\| \leq \varepsilon} \sigma(\|p\|) d p_{1} d p_{2}=1 .
$$

Consider a supported in $U_{i}$ closed 2 -form

$$
\eta_{i}=\sigma d p_{1} \wedge d p_{2}
$$

Note that the form

$$
\widetilde{\Omega}=\Omega+\sum_{1}^{N} a_{i} \eta_{i}
$$

is symplectic, as it follows from the explicit expressions (2) and (3). Note also that the restriction of the form $\eta_{i}$ to the fiber containing $\gamma_{i}$ vanishes, and hence for a sufficiently small $\varepsilon>0$ the form $\widetilde{\omega}=\left.\widetilde{\Omega}\right|_{V}$ is positive on the fibers of the 
fibration $V \rightarrow S^{1}$. Let us show that $\operatorname{Flux}\left(\operatorname{Hol}_{V, \omega} \circ \mathrm{Hol}_{V, \widetilde{\omega}}^{-1}\right)=a$. For any oriented curve $\gamma$ in $F_{0}$ we have

$$
\int_{\Gamma} \eta_{i}=D \gamma_{i}[\gamma] \text { and }\left.\omega\right|_{\Gamma}=0,
$$

where $\Gamma=\gamma \times l$. Let $\widetilde{\Gamma}$ be a cylinder formed by the characteristics of $-\widetilde{\omega}$ in $V_{1 / 2}$ originated at $\gamma \times 1 / 2$, and $\widehat{\Gamma}$ the projection of $\widetilde{\Gamma}$ to $F_{0}$. Note that $\int_{\widetilde{\Gamma}} \widetilde{\omega}=0$ and that the cylinders $\widehat{\Gamma}$ and $\widetilde{\Gamma}$ fit together into a cylinder with the same boundary as $\Gamma$. Let us orient $\Gamma, \widetilde{\Gamma}$ and $\widehat{\Gamma}$ in such a way that

$$
\partial \Gamma=\gamma \times 1 / 2-\gamma \times 0, \partial \widetilde{\Gamma}=\widetilde{\gamma}-\gamma, \partial \widehat{\Gamma}=\gamma \times 1 / 2-\widetilde{\gamma},
$$

where $\widetilde{\gamma}=\widetilde{\Gamma} \cap F_{0}$. The diffeomorphism $\mathrm{Hol}_{V, \omega} \circ \mathrm{Hol}_{V, \widetilde{\omega}}^{-1}$ coincides with the projection $F_{0} \rightarrow F_{0} \times 1 / 2$ followed by the holonomy along the characteristic foliation of $-\left.\widetilde{\omega}\right|_{V_{1 / 2}}$ Therefore,

$$
\begin{aligned}
\operatorname{Flux}\left(\operatorname{Hol}_{V, \omega} \circ \mathrm{Hol}_{V, \widetilde{\omega}}^{-1}\right)(\gamma) & =\int_{\widehat{\Gamma}} \omega=\int_{\widehat{\Gamma} \cup \widetilde{\Gamma}} \widetilde{\omega} \\
& =\int_{\Gamma} \widetilde{\omega}=\int_{\Gamma} \sum_{1}^{N} a_{i} \eta_{i}=\sum_{1}^{N} a_{i} D \gamma_{i}(\gamma)=a(\gamma) .
\end{aligned}
$$

To finish the proof of Theorem 3.1 it remains to fix the Hamiltonian isotopy class of the holonomy diffeomorphism. This can be done using the following standard argument from the theory of symplectic fibrations.

Lemma 3.5 Given any Hamiltonian diffeomorphism $h: F_{0} \rightarrow F_{0}$, consider a symplectic fibration $\left(V=F_{0} \times S^{1}, \omega\right)$ with $\mathrm{Hol}_{V, \omega}=h$. Then there exists a symplectic form $\Omega$ on $W=F_{0} \times D^{2}$ such that $\partial(W, \Omega)=(V, \omega)$.

Proof Let $H_{t}: F_{0} \rightarrow \mathbb{R}, t \in \mathbb{R}$, be a $2 \pi$-periodic time-dependant Hamiltonian whose time one map equals $h$. Suppose that $m<H_{t}<M$. We can assume that $m>0$. Consider an embedding $f: F_{0} \times S^{1} \rightarrow F_{0} \times \mathbb{R}^{2}$ given by the formula

$$
(x, t) \mapsto\left(x, \varphi=t, r=\sqrt{H_{t}(x)}\right),
$$

where $x \in F_{0}, t \in S^{1}=\mathbb{R} / 2 \pi$, and $(r, \varphi)$ are polar coordinate on $\mathbb{R}^{2}$. Let $\omega_{0}=$ $\left.\omega\right|_{F_{0}}$ and $\Omega_{0}$ denote the split symplectic form $\omega_{0}+d\left(r^{2} d \varphi\right)$. Then $f^{*} \Omega_{0}=\omega$. On the other hand, the embedding $f$ extends to an embedding $\tilde{f}: F \times D^{2} \rightarrow F \times \mathbb{R}^{2}$, and hence the form $\omega$ extends to a symplectic form $\Omega=\widetilde{f^{*}} \Omega_{0}$ on $D^{2} \times S^{1}$. 
This finishes off the proof of Theorem 3.1

Before proving Theorem[1.3 let us make a general remark on gluing of symplectic manifolds along their boundaries.

Remark 3.6 Let $\left(W_{1}, \Omega_{1}\right)$ and $\left(W_{2}, \Omega_{2}\right)$ be two symplectic manifolds, and $V_{1} \subset \partial W_{1}$ and $V_{2} \subset \partial W_{2}$ be components of their boundaries. Suppose we are given an orientation reversing diffeomorphism $f: V_{1} \rightarrow-V_{2}$ such that $f^{*} \Omega_{2}=\Omega_{1}$. Then the manifold $W=W_{1} \underset{f\left(V_{1}\right)=V_{2}}{\cup} W_{2}$ inherits a canonical, up to a Hamiltonian diffeomorphism, symplectic structure $\Omega$. Indeed, according to the symplectic neighborhood theorem the restriction $\left.\Omega_{i}\right|_{V_{i}}, i=1,2$, determines $\Omega_{i}$ on a neighborhood of $V_{i}$ in $W_{i}$ uniquely up to a symplectomorphism fixed on $V_{i}$.

Proof of Theorem 1.3 Let $(\widetilde{W}, \widetilde{\Omega})$ be a cobordism between $(-V, \omega)$ and a symplectic fibration $\left(V^{\prime}, \omega^{\prime}\right)$ which is provided by Theorem 1.1 and $(W, \Omega)$ be a symplectic manifold bounded by $\left(-V^{\prime}, \omega^{\prime}\right)$ which we constructed in Theorem 3.1. The required cobordism $\left(W^{\prime}, \Omega^{\prime}\right)$ we then obtain by gluing $(\widetilde{W}, \Omega)$ and $(W, \Omega)$ along their common boundary, see above Remark 3.6. Moreover, note that $H_{1}\left(W^{\prime} ; \mathbb{Z}\right)=H_{1}(W, \mathbb{Z})$. Hence, one can arrange that $H_{1}\left(W^{\prime} ; \mathbb{Z}\right)=0$.

Proof of Corollary 1.4 According to a theorem of Giroux (see 14]), any contact manifold $(V, \xi)$ admits an open book decomposition. Hence, for any symplectic form which is positive on $\xi$ we can use Theorem 1.3 to find a symplectic manifold $(W, \Omega)$ with $\partial(W, \Omega)=(-V, \omega)$. Attaching $(W, \Omega)$ to a nondesirable component (or components) of the boundary of a semi-filling we will transform it to a filling.

\section{An alternative proof of Theorem 3.1}

The following lemma of Kotschick and Morita (see [18]) gives an alternative proof of Theorem 3.1

Lemma 3.7 Let $\mathcal{D}$ be the group of symplectic (i.e. area and orientation preserving) transformations of a closed surface $\left(F_{0}, \sigma\right)$ where $\sigma$ is an area form

with $\int_{F_{0}} \sigma=1$. Then the commutator $[\mathcal{D}, \mathcal{D}]$ contains the identity component $\mathcal{D}_{0}$. If the genus of $F_{0}$ is $>2$ then the group $\mathcal{D}$ is perfect, i.e. $\mathcal{D}=[\mathcal{D}, \mathcal{D}]$. 
The proof of this lemma is based on Banyaga's theorem [3] which states that $\left[\mathcal{D}_{0}, \mathcal{D}_{0}\right]=\mathcal{D}_{H}$, a theorem of Harer (see [17]) that the group

$$
H_{1}\left(\Gamma_{g}\right)=\Gamma_{g} /\left[\Gamma_{g}, \Gamma_{g}\right]
$$

where $\Gamma_{g}$ is the mapping class group of the surface of genus $g$, is trivial if $g>2$ (and it is finite for $g \leq 2$ ), and the following formula of Lalonde and Polterovich from [21. For any symplectomorphism $g \in \mathcal{D}$ and any $f \in \mathcal{D}_{0}$ we have $[g, f]=g f g^{-1} f^{-1} \in \mathcal{D}_{0}$ and

$$
\operatorname{Flux}([g, f])=g^{*}(\operatorname{Flux}(f))-\operatorname{Flux}(f) .
$$

In particular, if the linear operator $g^{*}: H^{1}\left(F_{0}, \mathbb{R}\right) \rightarrow H^{1}\left(F_{0}, \mathbb{R}\right)$ has no eigenvalues $=1$ then the formula

$$
f \mapsto \operatorname{Flux}([g, f])
$$

defines a surjective map of $\mathcal{D}_{0}$ onto $H^{1}\left(F_{0}, \mathbb{R}\right)$ (or $H^{1}\left(F_{0}, \mathbb{R} / \mathbb{Z}\right)$ if $F_{0}$ is the torus). Clearly, there are a lot of diffeomorphisms $g$ with this property, and therefore one can represent any Hamiltonian isotopy class from $\mathcal{D}_{0}$ as a commutator of a fixed $g \in \mathcal{D}$ and a Hamiltonian diffeomorphism.

Lemma 3.7 allows us to extend any symplectic fibration over a circle whose fiber has genus $\geq 2$ to a symplectic fibration over a surface with boundary. The minimal genus of this surface is equal $1+m$, where $m$ is the minimal number of commutators needed to decompose the class of $\mathrm{Hol}_{V, \omega}$ in the mapping class group into a product of commutators. This gives an alternative proof of Theorem 3.1 for the case when genus $\left(F_{0}\right) \geq 2$. The genus restriction is not a serious obstruction for applications. However, it is unclear whether it is possible to improve this construction to accommodate the condition $H_{1}(W)=0$.

\section{Different flavors of symplectic fillings}

We conclude this article by summarizing the known relations between all existing notions of symplectic filling which were introduced in my earlier papers.

A contact manifold $(V, \xi)$ is called

(Weak) Weakly symplectically fillable if there exists a symplectic manifold $(W, \omega)$ with $\partial W=V$ and with $\left.\omega\right|_{\xi}>0$;

(Strong) Strongly symplectically fillable if there exists a symplectic manifold $(W, \omega)$ with $\partial W=V$ such that $\omega$ is exact near the boundary and there exists its primitive $\alpha$ such that $\xi=\left\{\left.\alpha\right|_{V}=0\right\}$ and $\left.d \alpha\right|_{\xi}>0$; 
(Stein-1) Stein (or Weinstein) fillable if it can be filled by Weinstein symplectic manifold, i.e. an exact symplectic manifold $(W, \omega)$ such that $\omega$ admits a primitive $\alpha$ such that the Liouville vector field $X$ which is $\omega$-dual to $\alpha$ (i.e. $X\lrcorner \omega=\alpha$ ) is gradient-like for a Morse function on $W$ which is constant and attains its maximum value on the boundary.

Stein fillability admits several equivalent reformulations. $(V, \xi)$ is Stein fillable if and only if

(Stein-2) $(V, \xi)$ can be obtained by a sequence of index 1 contact surgeries and index 2 surgeries along Legendrian knots with the $(-1)$-framing with respect to the framing given by the vector field normal to the contact structure;

(Stein-3) $(V, \xi)$ is compatible with an open book decomposition which arises on the boundary of a Lefschetz fibration over a disc such that the holonomy diffeomorphisms around singular fibers are positive Dehn twists;

(Stein-4) $(V, \xi)$ is holomorphically fillable i.e. there exists a complex manifold $W$ which has $V$ as its strictly pseudo-convex boundary and $\xi$ is realized as the fields complex tangencies to the boundary.

The equivalence of (Stein-1) and (Stein-2) follows from [9] or [29]. The equivalence between (Stein-2) and (Stein-3) is established in 1] and 24. The implication (Stein-1) $\Rightarrow($ Stein-4) is established in 9, while the opposite implication follows from [4].

Clearly,

$$
(\text { Stein }) \Rightarrow(\text { Strong }) \Rightarrow(\text { Weak })
$$

and all these notions imply the tightness, see [7] and [16]. As it is shown in this paper the notion of (weak/strong) semi-fillability introduced in [10] is equivalent to (weak/strong) fillability, and hence from now on it should disappear.

Here is a summary of what is known about the relation between three above notions of fillability and the notion of tightness.

Tightness does not imply weak fillability. Such an example was first constructed by John Etnyre and Ko Honda in [11. More examples were constructed by Paolo Lisca and András Stipsicz in [23].

Weak fillability does not imply strong fillability. For instance, it was shown in [8] that the contact structures $\xi_{n}$ on the 3-torus induced from the standard contact structure by a $n$-sheeted covering are all weakly symplectically fillable, but not strongly fillable if $n>1$. 
It is not known whether strong fillability implies Stein fillability. There are, however, examples of strong symplectic fillings which are not Stein fillings. The first example of this kind is due to Dusa McDuff from 25] who constructed an exact symplectic manifold $(W, \omega)$ with a disconnected contact boundary $\partial W=V_{1} \bigsqcup V_{2}$. This manifold cannot carry a Stein structure because it is not homotopy equivalent to a 2 -dimensional cell complex. Let us also point out that for $\operatorname{dim} V>3$ the notions of Stein and strong symplectic fillability do not coincide: using a modification of the above McDuff's argument one can construct a strongly fillable contact manifold which cannot be a boundary of a manifold homotopy equivalent to a half-dimensional cell complex.

We will finish this section by showing that one possible notion of fillability which seems to be intermediate between the conditions (Weak) and (Strong) is, in fact, equivalent to strong fillability. The Proposition 4.1 is equivalent to Lemma 3.1 in [6]. It also appeared in [26].

Proposition 4.1 Suppose that a symplectic manifold $(W, \omega)$ weakly fills a contact manifold $(V, \xi)$. Then if the form $\omega$ is exact near $\partial W=V$ then it can be modified into a symplectic form $\widetilde{\omega}$ such that $(W, \widetilde{\omega})$ is a strong symplectic filling of $(V, \xi)$.

Proof Let $\lambda$ be a contact form which defines $\xi$ such that $\left.d \lambda\right|_{\xi}=\left.\omega\right|_{\xi}$. According to Lemma 2.1 for a sufficiently small $\varepsilon>0$ and an arbitrarily large constant $C>0$ there exists a symplectic form $\Omega$ on $W$ which coincides with $\omega$ outside the $2 \varepsilon$-tubular neighborhood $U_{2 \varepsilon}$ of $\partial W$, and is equal to

$$
C d(t \lambda)+\omega, t \in[1-\varepsilon, 1],
$$

inside the $\varepsilon$-tubular neighborhood $U_{\varepsilon}$ of $\partial W$. By assumption, $\omega$ is exact near the boundary. Hence, we can assume that $\omega=d \alpha$ in $U_{\varepsilon}$. Let $\varphi$ be a cut-off function on $U_{\varepsilon}$ which is equal to 0 near $\partial W$, and is equal to 1 near the other component of the boundary of $\partial U_{\varepsilon}$. Then if $C$ is large the form

$$
\widetilde{\Omega}=C d(t \lambda)+d(\varphi \alpha)
$$

is symplectic, and together with $\Omega$ on $W \backslash U_{\varepsilon}$ defines a strong symplectic filling of $(V, \xi)$.

Remark 4.2 There are known several results concerning so-called concave symplectic fillings (which means $\partial(W, \omega)=(-V, \xi)$ ). Paolo Lisca and Gordana Matič proved in 22] that any Stein fillable contact manifolds embeds as a separating hypersurface of contact type into a closed symplectic manifold (in fact a 
complex projective manifold). Selman Akbulut and Burak Ozbagci gave in [2] a more constructive proof of this fact. Their construction topologically equivalent to one considered in this paper, though they did not considered the problem of extension of the taming symplectic form $\omega$. John Etnyre and Ko Honda showed (see [12]) that any contact manifold admits a concave symplectic filling which implies that a symplectic manifold which strongly fills a contact manifold can be realized as a domain in a closed symplectic manifold. A different proof of this result is given by David Gay in [13. Theorem 1.3 proven in this paper asserts a similar result for weak symplectic fillings. After learning about this article John Etnyre sent me an argument which shows that the weak case can be deduced from the strong one, thus giving an alternative proof of Theorem 1.3. His idea is that by performing a sequence of Legendrian contact surgeries it is possible to transform a contact manifold into a homology sphere and thus, taking into account an argument from Proposition 4.1. to reduce the problem to the case considered in their paper 12 with Ko Honda.

\section{References}

[1] S Akbulut, B Ozbagci, Lefschetz fibrations on compact Stein surfaces, Geom. Topol. 5 (2001) 319-334

[2] S Akbulut, B Ozbagci, On the topology of compact Stein surfaces, Int. Math. Res. Notices, 15 (2002) 769-780

[3] A Banyaga, Sur la structure du groupe de difféomorphismes qui préservent une forme symplectique, Comm. Math. Helvet. 53 (1978) 174-227

[4] F Bogomolov, B de Oliveira, Stein small deformations of strictly pseudoconvex surfaces, Contemp. Math. 207, 25-41

[5] E Calabi, On the group of automorphisms of a symplectic manifold, from: "Problems in Analysis", Princ. Univ. Press (1970) 1-26

[6] Y Eliashberg, On symplectic manifolds which some contact properties, J. of Diff. Geom. 33 (1991) 233-238

[7] Y Eliashberg, Filling by holomorphic discs and its applications, London Math. Soc. Lect. Notes 151 (1991) 45-68

[8] Y Eliashberg, Unique holomorphically fillable contact structure on the 3-torus, Int. Math. Res. Notices, 2 (1996) 77-82

[9] Y Eliashberg, Topological characterization of Stein manifolds of dimension $>$ 4, International Journal of Math. 1 (1990) 29-46

[10] Y Eliashberg, W P Thurston, Confoliations, University Lectures Series, AMS, 13 (1997) 
[11] J Etnyre, Ko Honda, Tight contact structures with no symplectic filling, Invent. Math. 148 (2002) 609-626

[12] J Etnyre, Ko Honda, On Symplectic Cobordisms, Math. Annalen, 323 (2002) 31-39

[13] D Gay, Explicit concave fillings of contact three-manifolds, Proc. Cam. Phil. Soc. 133 (2002) 431-441

[14] E Giroux, Géométrie de contact: de la dimension trois vers les dimensions supérieures, Proc. ICM-Beijing, 2 (2002) 405-414

[15] R Gompf, A topological characterization of symplectic manifolds, e-print arXiv:math.SG/0210103

[16] M Gromov, Pseudo-holomorphic curves in symplectic manifolds, Invent. Math. 82 (1985) 307-347

[17] J Harer, The second homology of the mapping class group of an orientable surface, Invent. Math. 72 (1983) 221-239

[18] D Kotschick, S Morita, Signatures of foliated surface bundles and the symplectomorphism groups of surfaces, arXiv:math.SG0305182

[19] P Kronheimer, T Mrowka, Monopoles and contact structures, Invent. Math. 130 (1997) 209-255

[20] $\mathbf{P}$ Kronheimer, T Mrowka, Witten's conjecture and Property $P$, Geom. Topol. 8 (2004) 295-310

[21] F Lalonde, L Polterovich, Symplectic diffeomorphisms as isometries of Hofer's norm, Topology, 36 (1997) 711-727

[22] P Lisca, G Matič, Tight contact structures and Seiberg-Witten invariants, Invent. Math. 129 (1997) $509-525$

[23] P Lisca, A Stipsicz, An infinite family of tight, not semi-fillable contact threemanifolds, arXiv:math.SG/0208063

[24] A Loi, R Piergallini, Compact Stein surfaces with boundary as branched covers of $B^{4}$, Invent. Math. 143 (2001) 325-348

[25] D McDuff, Symplectic manifolds with contact type boundaries, Invent. Math. 103 (1991) 651-671

[26] H Ohta, K Ono, Simple singularities and topology of symplectically filling 4-manifold, Comment. Math. Helv. 74 (1999) 575-590

[27] $\mathbf{P}$ Ozsváth, $\mathbf{Z}$ Szabó, Holomorphic discs and genus bounds, Geom. Topol. 8 (2004) 311-334

[28] W P Thurston, Some simple examples of symplectic manifolds, Proc. Amer. Math. Soc. 55 (1976) 467-468

[29] A Weinstein, Contact surgery and symplectic handlebodies, Hokkaido Math. J. 20 (1991) 241-251 